head of Basel, Switzerland-based Novartis's drug life-cycle management.

On their own, both GLP-1 agonists and basal insulin achieve impressive reductions of blood sugar levels. But with insulin use comes the risk of hypoglycemia and weight gain, and GLP-1 drugs can lead to nausea and weight loss. When the two are in combination, the weight loss seen with GLP-1 agonists offset the weight gain that is common with basal insulin, says Riccardo Perfetti, Sanofi's vice president of global medical affairs in the diabetes division. Patients typically experience less nausea with the
The question is: can they charge for two products instead of one?
Targeting this population could give the companies an edge in the highly competitive diabetes market. But for fixed-dose combinations to succeed, a company needs to price the drug lower than the individual components, according to Ronny Gal, a pharma analyst at Sanford Bernstein in New York City. "The question is: can they charge for two products instead of one?" Gal says, predicting US sales of $\$ 1.4$ billion in 2020 for IDegLira, with Novo's drug capturing $80 \%$ of the market, compared to $20 \%$ for Sanofi's iGlarLixi, according to Gal's research.

For Sanofi, combination, since the GLP-1 dose is lower in combination than when used as a single agent, and can be titrated.

The most salient benefit of combination drug therapy is greater compliance, as shown in a study combining aspirin, statins and two blood-pressure-lowering agents (JAMA 310, 918-929, 2013). If the drugs in question are injected, the adherence aspect is even more important, according to Novo Nordisk's Moses. As a consequence, in type 2 diabetes, the new long-acting, fixed-dose formulations, requiring a daily single injection, could be particularly beneficial for patients who have already been on insulin therapy or an GlP-1 agonist but failed to respond sufficiently. there is hope that the combination will be able to absorb some of the projected loss linked to the patent expiry on Lantus, its best-selling drug. Lantus, which made €6.4 (\$7.2) billion for the company last year, is set to see competition from biosimilars, such as Boehringer Ingelheim and Eli Lilly's Basaglar (insulin glargine), in the US by the end of 2016.

In the US, spending on type 2 diabetes drugs reached $\$ 43.9$ billion in 2015 , according to the IMS Institute's Medicines Use and Spending in the US report. The global diabetes market is also poised for growth. According to a recent report, approximately
150 million people have diabetes mellitus worldwide, and this number may well double by the year 2025, according to the World Health Organization.

Both companies view combinations as a way to persuade doctors to prescribe their drug to patients who have failed oral therapy, leapfrogging injectables such as insulin or GLP-1, potentially leading to a larger market share. "These are patients on oral agents who need more blood sugar control where it is fairly clear they will need a powerful medicine," says John Buse, who heads the division of endocrinology at the University of North Carolina School of Medicine.

Because the patients taking the combinations have higher rates of hypoglycemia than those taking GLP-1 agonists used alone, it may be more beneficial to use a GLP-1 drug first, says Nauck. The advantages of using GLP-1 receptor agonists for diabetes management have come to light recently. Not only do they reduce weight and Alc levels, but they may also improve cardiac parameters (Box 1).

Both IDegLira and iGlarLixi were reviewed by the FDA's Endocrinologic and Metabolic Drugs Advisory Committee in May. Sanofi used a priority review voucher (Nat. Biotechnol. 26, 1315-1316, 2008) to gain approval before its rival, but Novo now expects the FDA's decision first.

Eva von Schaper Munich, Germany

\title{
Farmers' microbe aid
}

Farmers have a handy new tool to test potential pathogens lurking in their soil. San Francisco-based startup Trace Genomics has launched a soil microbiome testing kit that uses genetic sequencing to identify and quantify disease-causing organisms to give farmers an insight into soil health before planting. Trace Genomics' first product, a \$199 Soil Pathogen Panel, tests for ten different microbes affecting strawberry and lettuce, including Verticillium, Fusarium, Macrophomina and Phytophthora (pictured). The turnaround time is about two weeks. Trace cofounders Poornima Parameswaran and Diane Wu, both from Andrew Z. Fire's laboratory, at Stanford University, say that their ultimate aim is to build a database containing 'fingerprints' from every farm on the planet to help growers prevent losses due to soil-borne disease.

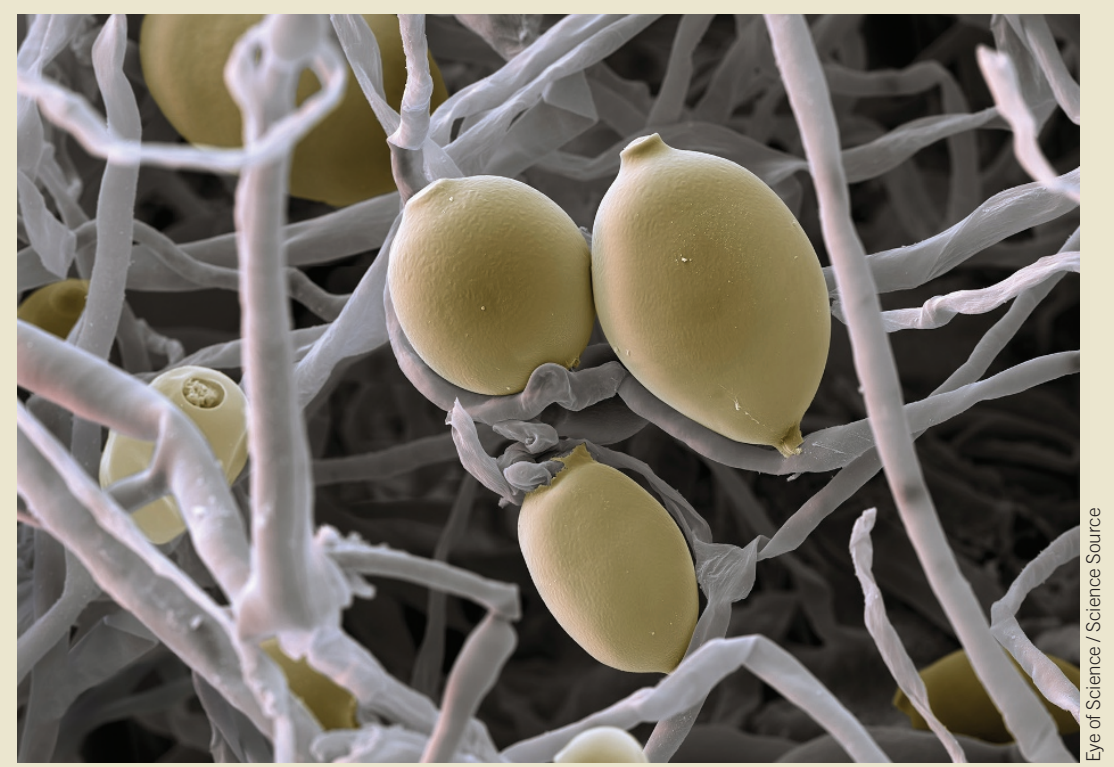

